\title{
Les ressources internationales en éducation
}

\section{Françoise Profit}

\section{(2) OpenEdition}

Journals

Édition électronique

URL : https://journals.openedition.org/ries/1654

DOI : 10.4000/ries. 1654

ISSN : 2261-4265

\section{Éditeur}

France Education international

\section{Édition imprimée}

Date de publication : 1 avril 2004

Pagination : 11-13

ISBN : 978-2-85420-560-2

ISSN : 1254-4590

\section{Référence électronique}

Françoise Profit, "Les ressources internationales en éducation », Revue internationale d'éducation de Sèvres [En ligne], 35 | avril 2004, mis en ligne le 11 avril 2012, consulté le 09 juillet 2021. URL : http:// journals.openedition.org/ries/1654; DOI : https://doi.org/10.4000/ries.1654

Ce document a été généré automatiquement le 9 juillet 2021

(c) Tous droits réservés 


\title{
Les ressources internationales en éducation
}

\author{
Françoise Profit
}

1 La rubrique Ressources en ligne est désormais consultable sur le site du CIEP : http:// www.ciep.fr/sitographie/index.htm

\section{Les ressources internationales en éducation}

2 Nous présentons ici un panorama non exhaustif de sources utiles dans le domaine de l'éducation, issues de sites français et étrangers. Il s'agit de sites d'informations générales, de portails et de bases de données qui permettent de s'orienter et de trouver rapidement une information synthétique et complète sur un pays dans le domaine éducatif, mais également au niveau linguistique, économique, politique et social. Nous proposons dans un premier temps une liste de sites transversaux qui offrent la possibilité de collecter des données dans de nombreux domaines, puis une sélection de sites consacrés à l'éducation, qui proposent souvent des études transversales et facilitent la comparaison des données.

\section{Données économiques, politiques et sociales pour tous les pays}

\section{Les Nations Unies}

Index des différents sites des organismes des Nations Unies, tous domaines confondus. La recherche peut être alphabétique ou thématique. http://www.unsystem.org/fr/ 


\section{Countries of the world}

4 Cette base de données fournit une fiche descriptive par pays contenant des informations d'ordre général pour chacun d'entre eux : cartes, éléments économiques, politiques et démographiques. http://www.infoplease.com/countries.html

\section{Library of Congress}

5 La bibliothèque du Congrès américain propose, pour tous les pays, une sélection de sites dans tous les domaines : généralités, commerce, culture, éducation, géographie, gouvernements, médias, etc. http://www.loc.gov/rr/international/portals.html

\section{Governements on the world wide web}

6 Base de données qui répertorie les sites des institutions des pays: ministères, parlements, ambassades par exemple. La recherche peut être effectuée par continent, par ordre alphabétique de pays et par type d'institution. Les informations n'ont pas été mises à jour depuis juin 2002 mais un grand nombre de liens restent valides. Site en anglais. http://www.gksoft.com/govt/en/

\section{Éducation}

\section{Organismes internationaux}

\section{L'Organisation de coopération et de développement économique (OCDE).}

7 Cet organisme fournit à ses trente pays membres des éléments statistiques et des publications traitant des politiques éducatives, permettant ainsi d'obtenir des informations transversales faisant le lien avec les politiques sociales et économiques. http://www.oecd.org/topic/0,2686,fr_2649_37455_1_1_1_1_37455,00.html

\section{La Banque mondiale}

8 Le site de la Banque mondiale est en anglais. Une présentation complète des différentes grandes rubriques concernant l'éducation est consultable sur une page de présentation : http://www1.worldbank.org/education/webguide.asp

9 Le site donne en particulier accès à une base de données de statistiques élaborée en partenariat avec l'OCDE et l'UNESCO. La recherche peut s'effectuer par pays, par thèmes principaux et par grandes régions. On peut également trouver des informations économiques et sociales et des liens vers d'autres ressources. http:// www1.worldbank.org/education/edstats/index.html

\section{L'UNESCO}

10 Outre le portail principal, nous indiquons ici les sites les plus représentatifs des différents organismes relevant de l'UNESCO et traitant de l'éducation. 


\section{Le portail « Éducation » de l'UNESCO}

11 Le site consacré à l'éducation aborde toutes les problématiques: droit à l'éducation, politiques éducatives, niveaux d'enseignement, types d'enseignement, intégration des technologies dans la pédagogie, éducation à la paix. Il aborde également la problématique de l'éducation en situation de crise et de reconstruction. http:// portal.unesco.org/education/ev.php?

URL_ID=15200\&URL_DO=DO_TOPIC\&URL_SECTION=201\&reload=1076343351 Une page donne accès aux six instituts dépendants directement de l'UNESCO et traitant de l'éducation: http://portal.unesco.org/education/ev.php? URL_ID=23025\&URL_DO=DO_TOPIC\&URL_SECTION=201

\section{L'Institut de statistiques de l'UNESCO}

12 Le site met en ligne les derniers indicateurs éducatifs, les nouvelles publications et les projets mis en œuvre dans ce secteur. On y trouve les chiffres actualisés de tous les pays de l'enseignement pré-primaire à l'enseignement supérieur. http:// www.uis.unesco.org/ev_fr.php?ID=2867_201\&ID2=DO_TOPIC

Les données recueillies sont basées sur la Classification internationale type de l'éducation (CITE 97) : ce système de classification est un cadre polyvalent qui permet aux pays de comparer leurs progrès. http://stats.uis.unesco.org/fra/ReportFolders/Rfview/ explorerp.asp

\section{Le Bureau International d'Éducation (BIE)}

Le site offre une grande richesse de banques de données : informations sur les pays, sur les innovations, données mondiales en éducation, données sur l'éducation préventive et base de données bibliographiques. http://www.ibe.unesco.org Le site propose également ses publications et la possibilité d'acheter en ligne la revue Prospect. http:// www.ibe.unesco.org/International/Publications/Prospects/proshome.htm

\section{L'Institut International de Planification de l'Éducation (IIPE)}

15 L'IIPE est un centre de recherche et de formation qui tend à améliorer la qualité des systèmes éducatifs. De nombreuses publications sont accessibles ainsi que les informations concernant les axes de recherche pour l'éducation de base, l'enseignement supérieur, la qualité et la gestion de l'éducation. http:// www.unesco.org/iiep/fre/indexfr.htm

\section{Éducation pour tous}

Le site suit le processus "Éducation pour tous" engagé à Dakar en 2000¹. Les documents de référence, le cadre d'action sont disponibles, les rapports de suivi de 2001 à 2003 peuvent être consultés. Des forums régionaux sont également en ligne ainsi que les programmes-phares. http://www.unesco.org/education/efa/fr/index.shtml 


\section{Au niveau européen}

\section{Union européenne} en éducation, la politique européenne dans ce domaine, le compte-rendu des conférences et les liens vers les partenaires (ministères de l'éducation des pays d'Europe, organisations internationales, ONG). http://www.coe.int/T/F/ Coopération_culturelle/education/

\section{France}

\section{Europedu}

Site portail du ministère de l'Éducation nationale français, consacré à la création d'un enseignement supérieur en Europe, il regroupe les informations sur le processus de Bologne initié à Paris en 1998. Le site offre la liste des pays signataires, les évolutions envisagées, des informations générales et des descriptifs des systèmes d'enseignement supérieur par pays ainsi qu'un descriptif succinct des diplômes. Le site est en anglais, allemand, français et italien. http://www.sup.adc.education.fr/europedu/french/ 


\section{Atlas des sites}

Conçu par la direction des Relations internationales et de la coopération du ministère français de la Jeunesse, de l'Éducation nationale et de la Recherche, l'atlas des sites recense, à travers un planisphère, les informations éducatives disponibles sur le web. http://www.education.gouv.fr/int/atlas/default.htm

\section{NOTES}

1. Voir la description détaillée de ce site dans la Revue internationale d'éducation de Sèvres $\mathrm{n}^{\circ} 27$, octobre 2000, pp. 21-23.

INDEX

Mots-clés : organisation internationale, ressource documentaire, statistiques

\section{AUTEUR}

\section{FRANÇOISE PROFIT}

Documentaliste, centre de ressources documentaires du CIEP 